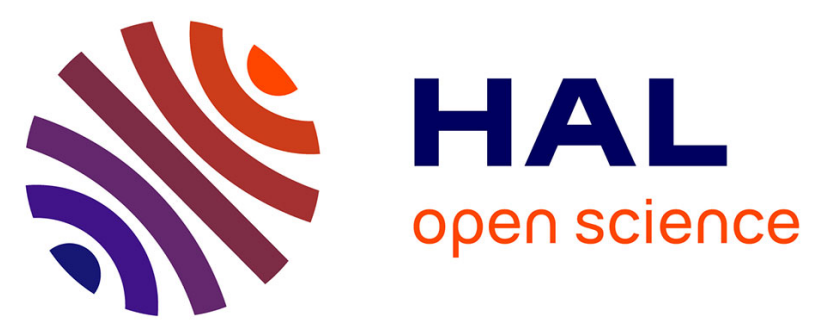

\title{
Efficient frequency-domain MMSE turbo equalization derivation and performance comparison with the time-domain counterpart
}

\author{
Karine Amis Cavalec, Nicolas Le Josse, Christophe Laot
}

\section{To cite this version:}

Karine Amis Cavalec, Nicolas Le Josse, Christophe Laot. Efficient frequency-domain MMSE turbo equalization derivation and performance comparison with the time-domain counterpart. ICWMC'07: proceedings of the third international conference on wireless and mobile communications, 4-7 mars, Guadeloupe, France, Mar 2007, Guadeloupe, France. pp.65 -, 10.1109/ICWMC.2007.38 . hal02121663

\section{HAL Id: hal-02121663 \\ https://hal.science/hal-02121663}

Submitted on 11 Jun 2021

HAL is a multi-disciplinary open access archive for the deposit and dissemination of scientific research documents, whether they are published or not. The documents may come from teaching and research institutions in France or abroad, or from public or private research centers.
L'archive ouverte pluridisciplinaire HAL, est destinée au dépôt et à la diffusion de documents scientifiques de niveau recherche, publiés ou non, émanant des établissements d'enseignement et de recherche français ou étrangers, des laboratoires publics ou privés. 


\title{
Efficient Frequency-Domain MMSE turbo equalization derivation and performance comparison with the Time-Domain counterpart
}

\author{
Karine Amis, Nicolas Le Josse and Christophe Laot \\ GET/ENST Bretagne, Signal and Communication Dept./TAMCIC (CNRS UMR 2872) \\ Technopôle Brest-Iroise, CS 83818, 29238 Brest Cedex 3, France \\ Email: karine.amis@enst-bretagne.fr, nicolas.lejosse@enst-bretagne.fr, christophe.laot@enst-bretagne.fr
}

\begin{abstract}
This paper addresses a novel frequency-domain (FD) derivation of the Minimum Mean Square Error (MMSE) Turbo Equalizer for ST-BICM systems. The receiver consists of a FD MMSE Equalizer and a Time Domain (TD) decoder with iterative information exchange between the two functions. In particular, we propose a FD MMSE equalizer derivation improved from previous approaches, in that feedback and feedforward filters are directly derived in the frequency-domain. The accuracy of the proposed approach is confirmed by comparing the lower bound of the iterative equalizing process with the corresponding analytical results proposed in previous paper. Simulations over multipath Rayleigh block fading channels show that the resulting algorithm exhibits similar performance with that of the corresponding TD MMSE equalization. The problem of finding the TD filter achieving the best compromise between minimum impulse response length and performance is considered.
\end{abstract}

\section{Introduction}

Wireless digital communications are about to take the place of wired applications. To overcome the need for an increasing volume of information through a band limited transmission medium, a solution is to use multiple antennas at both sides of the communication link. Space Time Bit Interleaved Modulations [13] have thus been proposed to achieve high spectral efficiency transmissions over frequency selective channels. Bit interleaved Modulation consists in cascading an information binary digit encoder, an interleaver and a modulator. For ST-BICM, modulated symbols are spatially splitted into $n_{T}$ substreams, where $n_{T}$ denotes the transmit antenna number. Each substream is sent from its corresponding antenna. Provided the channel is frequency selective, such a transmission scheme combines spatial, frequency and time diversities. The receiver has to be thought so as to take fully advantage of the available diversity. The Maximum Likelihood A Posteriori receiver achieves this goal but its computational cost is too high and makes its implementation impossible. The MMSE turbo equalization principle $[6,16,15]$ enables to derive low complexity receivers that can achieve optimal performance. It has been extended and applied to detect single carrier STBICM. In $[4,17,1]$, the MMSE equalization is carried out in the time domain.

Nevertheless, frequency-domain processing has retained more attention to be an effective mean for reducing computational complexity. Frequency domain approaches for MMSE turbo equalization are proposed in [14][7][3] for SISO and MIMO systems respectively. More precisely, The filtering equations, derived in the time-domain, are converted to the frequency-domain by taking advantage of the circular convolution property of the channel impulse response and the data symbols enabled by inserting a cyclic prefix at the beginning of each transmitted packet. The resulting structure includes only one filter in succession of a soft interference canceller.

In [8], the authors propose an interesting frequencydomain equation derivation for an iterative BLAST receiver. In the spirit of [8] and based on the approach of [10], we derive a Turbo equalizer for ST-BICM by optimizing the feedback and feedforward filters directly in the frequencydomain. In that sense, this paper improves the derivation of the previous approach [14][7][3].

In systems that communicate over large delay spread channels, it is well understood that TD MMSE turbo equalization provides comparable performance when configured with a high impulse response length filter. However a performance comparison between the frequency-domain MMSE turbo equalizer and its time-domain counterpart, for which the filter length has to be properly and efficiently chosen, has never been proposed yet. In this paper, we consider

\footnotetext{
This work was supported in part by FT RD.
} 
the problem of finding the TD filter achieving the best compromise between minimum impulse response length and performance.

In section II, we describe the MIMO transmission scheme and particularly the frame structure adaptation to frequency domain equalization. In section III, the turbo equalizer equations are derived and in section IV, simulation results show the receiver efficiency in comparison with that of the corresponding MMSE equalizer in the time-domain. In this section the TD filter length choice is pointed out.

\section{MIMO system description}

\subsection{Notations}

In the following, considering a matrix $\mathrm{A}, \mathrm{A}_{,, i}$ denotes the $i^{\text {th }}$ column of $\mathrm{A}$ and $\mathrm{A}_{j}$, denotes the $j^{\text {th }}$ row of $\mathrm{A} . \mathrm{A}^{\mathrm{T}}$ is the transpose of $\mathrm{A}$ and $\mathrm{A}^{\dagger}$ is the Hermitian transpose of $\mathrm{A}$. If $\{x(n)\}$ is a symbol sequence of size $N,\{X(p)\}$ denotes its $N$-Discrete Fourier Transform.

\subsection{Transmission model}

We consider a MIMO system consisting of $n_{T}$ transmit antennas and $n_{R}$ receive antennas. Information binary digits are first encoded thanks to a non recursive convolutional code before being interleaved. Interleaved coded bits are then modulated and passed through a serial to parallel converter. Each of the $n_{T}$ resulting symbol streams is sent from one of the $n_{T}$ transmit antennas. We denote $s_{i}(p)$ the $p^{\text {th }}$ symbol transmitted from the $i^{\text {th }}$ transmit antenna. The modulation size is denoted $M$ and $m$ is defined as $m=\log _{2}(M)$.

In case of a frequency selective channel, the received data result from the convolution of the channel impulse response and the data symbols. Frequency-domain receiver requires that the time domain convolution of the two finitelength sequences be equivalent to the product of their Discrete Fourier Transform coefficients. This is true only if the transmitted frame structure enables the convolution to be circular. This is achieved by inserting a cyclic prefix (the last $\nu$ symbols are reported at the beginning of the sequence) or extending each finite-length sequence by a known word [2]. In this paper, each substream symbols are splitted into packets of size $N$ and a cyclic prefix of size $\nu$ is inserted at the beginning of each packet. At the receiver side, the cyclic prefix is suppressed before operating the $N$ DFT.

Let us denote $\mathrm{h}_{j, i}(z)=\sum_{l=0}^{L-1} h_{j, i}(l) z^{-l}$ the Ztransform of the channel impulse response between transmit antenna $i$ and receive antenna $j$. The complex AWGN noise coefficients on receive antenna $j$ are denoted $b_{j}(n)$. Assum- ing perfect carrier and time synchronisation, the samples received on receive antenna $j$ can be written as follows.

$$
r_{j}(n)=\sum_{i=1}^{n_{T}} \sum_{l=0}^{L-1} h_{j, i}(l) s_{i}(n-l)+b_{j}(n)
$$

After the cyclic prefix extraction, $\left\{r_{j}(n)\right\}$ is splitted into packets of length $N$. A $N$ - DFT is applied on each block. We thus obtain $N$ vectors $\mathrm{R}(p)$ of size $n_{R}$ whose expression is

$$
\mathrm{R}(p)=\mathrm{H}(p) \mathrm{S}(p)+\mathrm{B}(p)
$$

where $\mathrm{R}(p)=\left[\begin{array}{lll}R_{1}(p) & \cdots & R_{n_{R}}(p)\end{array}\right]^{\mathrm{T}}, \quad \mathrm{S}(p)=$ $\left[\begin{array}{lll}S_{1}(p) & \cdots & S_{n_{T}}(p)\end{array}\right]^{\mathrm{T}}, \mathrm{H}(p)=\left[H_{j, i}(p)\right]_{\substack{1 \leq i \leq n_{T} \\ 1 \leq j \leq n_{R}}}$ with $\left\{H_{j, i}(p)\right\}$ the $N$-DFT of $\left\{h_{j, i}(l)\right\}$.

\subsection{Statistical property assumptions}

We assume that the interleaver depth is sufficient to ensure that information binary digits entering the modulator are independent and identically distributed. Denoting them $\alpha_{k}$, an additional hypothesis is $\operatorname{Pr}\left(\alpha_{k}=1\right)=\frac{1}{2}$. Taking into account a QAM or PSK modulation, the modulated symbols $s_{i}(n)$ have zero mean and the cross-correlation matrix coefficients are given by

$$
\mathrm{E}\left[s_{i}(n) s_{q}^{\star}(n-k)\right]=\sigma_{s}^{2} \delta_{k, 0} \delta_{i-q, 0}
$$

where $\delta_{k, 0}$ is the Kronecker symbol.

Complex noise samples are supposed to be independent, identically distributed of zero mean and variance $\sigma_{b}^{2}$.

\section{Frequency-Domain Turbo Equalization}

The turbo equalization principle [5] consists in repeating the equalization and decoding processes by exploiting information available at the decoder output in the equalizer. In the proposed receiver scheme, the equalization is operated in the frequency domain whereas the decoding process is carried out in the time domain.

\subsection{Information exchange principle}

The decoder provides the equalizer with A Posteriori Probabilities for the encoded binary digits that can be used to compute a soft estimate of transmitted symbols. We denote $L_{\text {Out }}^{D}(q)$ the Logarithm of Likelihood Ratio for the $q^{\text {th }}$ encoded bit available at the decoder output. $\left\{L_{\text {Out }}^{D}(q)\right\}$ are interleaved and provided to the equalizer input to estimate the Inter Symbol Interference (Co and Inter Antenna) and take it into account in the decision value computation. Let us denote $\tilde{s}_{i}(n)$ a soft estimate of $s_{i}(n)$. The samples $\tilde{s}_{i}(n)$ are computed using the regression curve theory, 
i.e. taking the conditional expectation. More precisely, $\tilde{s}_{i}(n)=\mathrm{E}\left[s_{i}(n) \mid\left\{L_{\operatorname{In}}^{E}(q)\right\}\right]$, where $\left\{L_{\operatorname{In}}^{E}(q)\right\}$ corresponds to the interleaving of $\left\{L_{\text {Out }}^{D}(q)\right\}$. Moreover as in [10], $\tilde{s}_{i}(n)$ are assumed to be independent and identically distributed of zero mean and variance denoted $\sigma_{\tilde{s}}^{2}$.

If $\mathcal{S}$ denotes the modulation constellation, using the bit to symbol conversion

$$
\left\{\alpha_{i}(t)\right\}_{m n \leq t \leq m(n+1)-1} \in\{0,1\}^{m} \leftrightarrow s_{i}(n) \in \mathcal{S},
$$

we can write

$$
\begin{aligned}
\tilde{s}_{i}(n) & =\sum_{s \in \mathcal{S}} s \operatorname{Pr}\left(s_{i}(n)=s \mid\left\{L_{\operatorname{In}}^{E}(q)\right\}\right) \\
& =\sum_{\substack{s \in \mathcal{S} \\
\left\{\alpha_{k}\right\} \leftrightarrow s}} s \prod_{t=m n}^{m(n+1)-1} \operatorname{Pr}\left(\alpha_{i}(t)=\alpha_{k} \mid\left\{L_{\operatorname{In}}^{E}(q)\right\}()\right.
\end{aligned}
$$

with

$\operatorname{Pr}\left(\alpha_{i}(k)=\alpha \mid\left\{L_{\operatorname{In}}^{E}(q)\right\}\right)=\frac{1}{2}\left(1+(2 \alpha-1) \tanh \left(\frac{L_{\operatorname{In}}^{E}(t)}{2}\right)\right)$.

where $\alpha_{i}(k)$ corresponds to the $t^{\text {th }}$ binary digit in the interleaved coded binary sequence. One can prove [10] that

$$
\begin{aligned}
& \mathrm{E}\left[s_{i}(n) \tilde{s}_{q}^{\star}(n-k)\right]=\sigma_{\tilde{s}}^{2} \delta_{k, 0} \delta_{i-q, 0} \\
& \mathrm{E}\left[\tilde{s}_{i}(n) \tilde{s}_{q}^{\star}(n-k)\right]=\sigma_{\tilde{s}}^{2} \delta_{k, 0} \delta_{i-q, 0}
\end{aligned}
$$

Both properties are used in the equalizer equation derivation.

\subsection{Frequency domain equalizer equation derivation}

Based on the approach in [6], the proposed equalizer consists of a feedforward and feedback MMSE filters in the frequency domain. We denote $\left\{\tilde{S}_{i}(p)\right\}$ the $N$-DFT of $\left\{\tilde{s}_{i}(n)\right\}$. The equalizer computes $\hat{S}_{i}(p)$ from $\mathrm{R}(p)$ and $\left\{\tilde{S}_{q}(p)\right\}_{1 \leq q \leq n_{T}}$. More explicitly, $\hat{S}_{i}(p)$ is given by

$$
\hat{S}_{i}(p)=\mathrm{W}_{i, \cdot}(p) \mathrm{R}(p)-\mathrm{Q}_{i, \cdot}(p) \tilde{\mathrm{S}}(p)
$$

where $\mathrm{W}_{i, .}(p)$ denotes the $n_{R}$ length row vector of the $p$ th feedforward filter frequency coefficients and $Q_{i, .}(p)$ is the $n_{T}$ length row vector of the $p$ th feedback filter coefficients such that its diagonal elements in the time domain is equal to zero: $q_{i, i}(0)=0$, i.e. $\sum_{p=0}^{N-1} Q_{i, i}(p)=0$.

To obtain the matrices $\mathrm{W}(p)$ and $\mathrm{Q}(p)$, we minimize the mean square error between $\hat{\mathrm{S}}(p)$ and $\mathrm{S}(p)$ under the constraint $\sum_{p=0}^{N-1} Q_{i, i}(p)=0$ for each index $i$. The Lagrangian resolution of this optimization problem provides us with the following equations :

$$
\mathrm{G}(p)=\left(1-\frac{\sigma_{\tilde{s}}^{2}}{\sigma_{s}^{2}}\right) \sum_{q=1}^{n_{T}} \mathrm{H}_{\cdot, q}(p)\left[\mathrm{H}_{\cdot, q}(p)\right]^{\dagger}+\frac{\sigma_{b}^{2}}{\sigma_{s}^{2}} \mathrm{I}_{h\left(\ell_{R} 0\right)}
$$

$$
\begin{aligned}
& \beta_{i}=\frac{1}{N} \sum_{p=0}^{N-1}\left[\mathrm{H}_{\cdot, i}(p)\right]^{\dagger}[\mathrm{G}(p)]^{-1} \mathrm{H}_{\cdot, i}(p) \\
& \mu_{i}=\frac{\beta_{i}}{1+\frac{\sigma_{\tilde{s}}^{2}}{\sigma_{s}^{2}} \beta_{i}} \\
& \mathrm{~W}_{i, \cdot}(p)=\left(1-\frac{\sigma_{\tilde{s}}^{2}}{\sigma_{s}^{2}} \mu_{i}\right)\left[\mathrm{H}_{i, \cdot}(p)\right]^{\dagger}[\mathrm{G}(p)]^{-1} \\
& \mathrm{Q}_{i, \cdot}(p)=\mathrm{W}_{i, \cdot}(p) \mathrm{H}(p)-\mu_{i} \mathrm{e}_{i}
\end{aligned}
$$

where $e_{i}$ is a $n_{T}$ length vector with coefficient in position $i$ equal to one and the other ones equal to zero. In $[1,13,15,16,18]$, estimation of $\mathrm{s}(n)$ is based on MMSE linear estimation requiring the computation of second order statistics at each signalling instant using the information delivered by the decoder. This approach provides a more accurate estimation of $\mathrm{s}(n)$ at the expense of a higher complexity $[10,15]$. Note that, after some mathematical developpements, we can obtain an equalizer structure equivalent to that of [3][7], i.e a soft interference canceler followed by a MMSE linear filter in frequency-domain.

\subsection{Analysis of the equalizer output}

Considering the expression of $\mathrm{R}(p)$ and equations (1014), one can easily obtain an equivalent expression of the equalizer output :

$$
\hat{S}_{i}(p)=\mu_{i} S_{i}(p)+\mathrm{Q}_{i, \cdot}(p)(\mathrm{S}(p)-\tilde{\mathrm{S}}(p))+\mathrm{W}_{i, \cdot}(p) \mathrm{B}(p)
$$

Therefore one can write $\hat{s}_{i}(n)=\mu_{i} s_{i}(n)+\eta(n)$ where $\eta(n)$ corresponds to the residual ISI and the noise. Indeed due to the constraint on $\left\{\mathrm{Q}_{i, i}(p)\right\}, \eta(n)$ and $s_{i}(n)$ are independent. We assume that $\eta(n)$ is a complex gaussian noise. We can prove that $\eta(n)$ is a zero mean random variable of variance $\sigma_{\eta}^{2}$ equal to $\sigma_{s}^{2} \mu_{i}\left(1-\mu_{i}\right)$. Therefore a direct approximation of the conditional density probability function of $\hat{s}_{i}(n)$ is

$$
p_{\hat{s}_{i}(n) \mid s_{i}(n)}(x)=\frac{1}{\pi \sigma_{\eta}^{2}} \exp \left(-\frac{\left|x-\mu_{i} s_{i}(n)\right|^{2}}{\sigma_{\eta}^{2}}\right)
$$

\subsection{Soft symbol to binary digit conversion}

We define the extrinsic information delivered by the decoder by $\mathrm{X}_{\text {Out }}^{D}=\mathrm{L}_{\text {Out }}^{D}-\mathrm{X}_{\mathrm{In}}^{D} \cdot X_{\text {Out }}^{D}(q)$ is independent from $X_{\operatorname{In}}^{D}(q)$ which is delivered by the equalizer. Therefore it will be used as a valuable a priori piece of information in the soft symbol to binary digit converter. We denote $\mathrm{X}_{\mathrm{In}}^{E}$ the interleaved sequence of $\mathrm{X}_{\mathrm{Out}}^{D}$. Assuming that $\alpha_{i}(m n+k)$ corresponds to the position $q$ in the serial binary stream (i.e before the modulation and the serial to parallel conversion), the Logarithm of Likelihood Ratio for the $q^{\text {th }}$ binary digit 
is given by :

$L_{\text {Out }}^{E}(q)=\ln \left(\frac{\sum_{s \in \mathcal{S} \mid \alpha_{k}=1} p_{\hat{s}_{i}(n) \mid s_{i}(n)}(x) \operatorname{Pr}\left(s_{i}(n)=s \mid \mathrm{X}_{\text {In }}^{E}\right)}{\sum_{s \in \mathcal{S} \mid \alpha_{k}=0} p_{\hat{s}_{i}(n) \mid s_{i}(n)}(x) \operatorname{Pr}\left(s_{i}(n)=s \mid \mathrm{X}_{\text {In }}^{E}\right)}\right)$

The symbol to binary digit converter output is defined as the extrinsic information extracted from $L_{\text {Out }}^{E}(q)$ and independent of $X_{\operatorname{In}}^{E}(q)$.

$$
X_{\text {Out }}^{E}(q)=L_{\text {Out }}^{E}(q)-X_{\mathrm{In}}^{E}(q)
$$

$\mathrm{X}_{\text {Out }}^{E}$ is desinterleaved giving $\mathrm{X}_{\mathrm{In}}^{D}$. $\mathrm{X}_{\mathrm{In}}^{D}$ becomes the new decoder input.

The whole receiver structure is summarized in Fig.1.

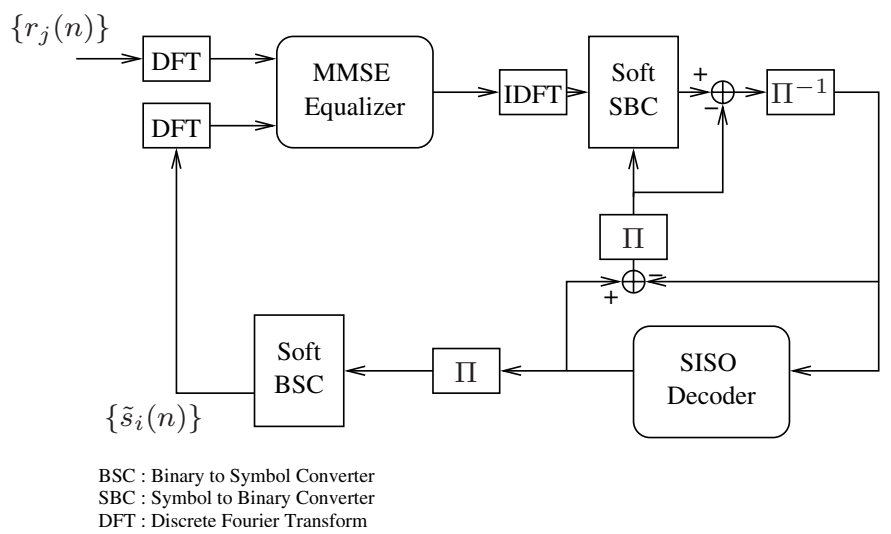

Figure 1. Frequency domain turbo equalizer structure

\subsection{Lower bound of the turbo equalizer performance}

A lower bound of the iterative equalizing and decoding process can be obtained by feeding the equalizer with perfect decoder output. It consists in considering $\tilde{\mathrm{S}}(p)$ equal to $\mathrm{S}(p)$ in (9) and taking $X_{\mathrm{In}}^{E}(q)=\gamma\left[2 \alpha_{i}(m n+k)-1\right]$ where $\alpha_{i}(m n+k)$ corresponds to the $q^{\text {th }}$ binary digit in the interleaved coded sequence and $\gamma$ is a constant of high value. The so-called genie FD MMSE turbo equalizer supresses multi-antenna interference and intersymbol interference for each transmit antenna, from the received signal. Assuming soft demapping without a priori knowledge, we can accurately predict asymptotic performance at the frequencydomain equalizer output following the proposed approach in [11]. Simulations are shown in section IV to confirm the purpose.

\section{Simulation results}

This section is dedicated to the simulation result presentation. Before introducing the parameters, we define the Signal to Noise Ratio and its expression as a function of $\frac{E_{b}}{N_{0}}$. $E_{b}$ is the average energy necessary to transmit an information binary digit and $N_{0}$ is the unilateral power spectral density function of the gaussian noise.

\subsection{Signal to Noise Ratio definition}

According to (3), the average total transmitted power per channel use is given by $P_{s}=n_{T} \sigma_{s}^{2}$. Defining a super symbol as $\mathrm{s}(n)=\left[s_{1}(n) \cdots s_{n_{T}}(n)\right]^{\mathrm{T}}$, and its duration $T$, and denoting $E_{s}$ the average energy necessary to transmit a super symbol, we can write $E_{s}=P_{s} T$. On the other hand, if $R_{c}$ is the coding rate, the following equality holds: $E_{s}=n_{T} m R_{c} E_{b}$. If the reception low-pass filter is the square root of a raised cosinus filter, the average gaussian noise power is equal to $P_{b}=\frac{N_{0}}{T}$. Furthermore $P_{b}=\sigma_{b}^{2}$. As a consequence, the average signal to noise ratio per receive antenna is

$$
\rho_{j}=\mathrm{E}\left[\sum_{i=1}^{n_{T}}\left\|\mathrm{~h}_{j i}\right\|^{2}\right] \frac{P_{s}}{n_{T} P_{b}}=m R_{c} \frac{E_{b}}{N_{0}} \mathrm{E}\left[\sum_{i=1}^{n_{T}}\left\|\mathrm{~h}_{j i}\right\|^{2}\right]
$$

where $\left\|\mathrm{h}_{j i}\right\|^{2}=\sum_{l=0}^{L-1}\left|h_{j i}(l)\right|^{2}$.

\subsection{Turbo equalizer performance over an ISI channel}

The simulation context is the same as in [4]. We consider a MIMO system with $n_{T}=n_{R}=2$. The information sequence length is 506 . The convolutional code has a coding rate of $\frac{1}{3}$ and its octal polynomial representation is $(133,145,175)$. Binary coded digits are pseudo-randomly interleaved before being 8-PSK modulated. We consider that the interleaver depth is sufficient to ensure information binary digits entering the modulator are i.i.d. Simulations have shown that taking higher interleaver size does not improve the bit error rate. Then symbols are splitted into packets of size 256 . A cyclic prefix is added at the beginning of each packet. We assume that channel impulse responses between two transmit and receive antenna pairs are uncorrelated and consist of two paths separated by a symbol duration and of equal average power. $\mathrm{h}_{j i}(z)=$ $h_{j i}(0)+h_{j i}(1) z^{-1}$ with $h_{j i}(l) \sim \mathcal{N}\left(0, \frac{1}{4}\right)+j \mathcal{N}\left(0, \frac{1}{4}\right)$. The channel is time-invariant over a symbol packet duration (i.e over $N+\nu$ symbols) and we assume perfect channel state information at the receiver. Assuming soft demapping without a priori knowledge, the bit error rate (resp. Frame error rate) after each equalization and decoding iteration is plotted as a function of $\frac{E_{b}}{N_{0}}$ in Fig. 4.2 (resp. 


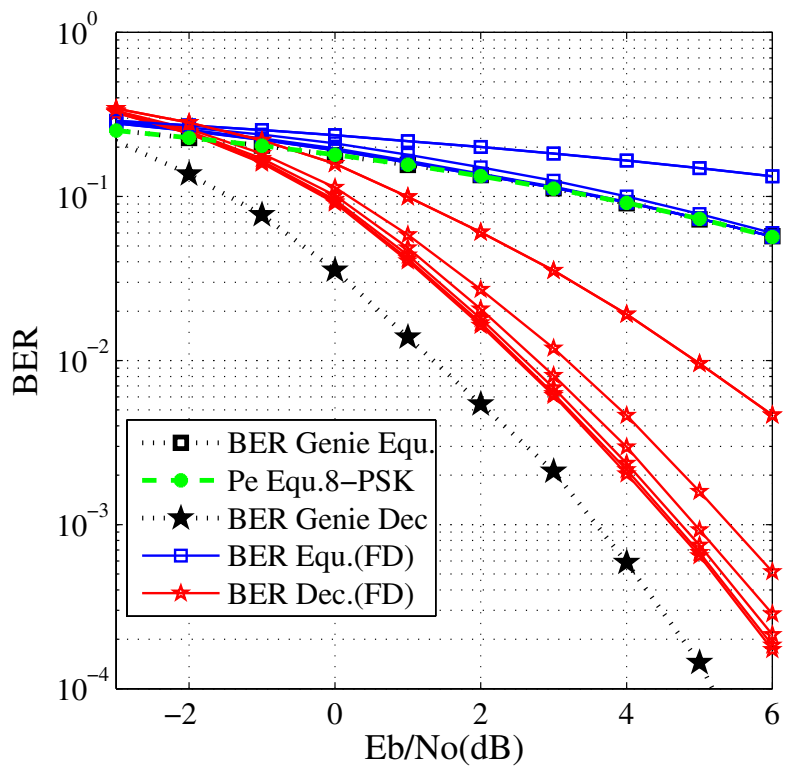

Figure 2. BER as a function of $\frac{E_{b}}{N_{0}}-2 \mathrm{Tx} 2 \mathrm{Rx}$
- CC(133,145,175)- 8-PSK

Fig. 3). The signal to noise ratio computation does not take into account the cyclic prefix and the trellis termination bits. The performance of the turbo equalizer with perfect a priori information from the decoder (cf. III.E) is plotted as a reference (genie-equalizer and genie-decoder). The genieequalizer performance is compared to the corresponding analytical probability of bit error developed in [11]. We observe that both curves are indistinguishable which confirm the accuracy of the proposed FD algorithm. The figure also shows that for $\frac{E_{b}}{N_{0}}$ superior to $2 \mathrm{~dB}$, the iterative process at the equalizer output reaches a steady-state after 3 iterations which matches the lower bound. On the other hand, for a BER of $10^{-2}$ at the decoder output, there is a gap of 1.5 $\mathrm{dB}$ compared to the genie-aided decoder output. This is due to the fact that the BER at the equalizer output is not exactly the same as the one available at the genie-aided output and this little difference is amplified at the decoder output. Indeed the decoder is very sensitive to the reliability value level. In Fig.3, the behaviour for FER is somehow different. The decoder performance after 6 iterations is at less than 0.4 $\mathrm{dB}$ from the genie-aided decoder. Considering the iterative receiver convergence, we can observe that 4 iterations are sufficient. The improvement brought by the fifth and sixth iterations is negligible.

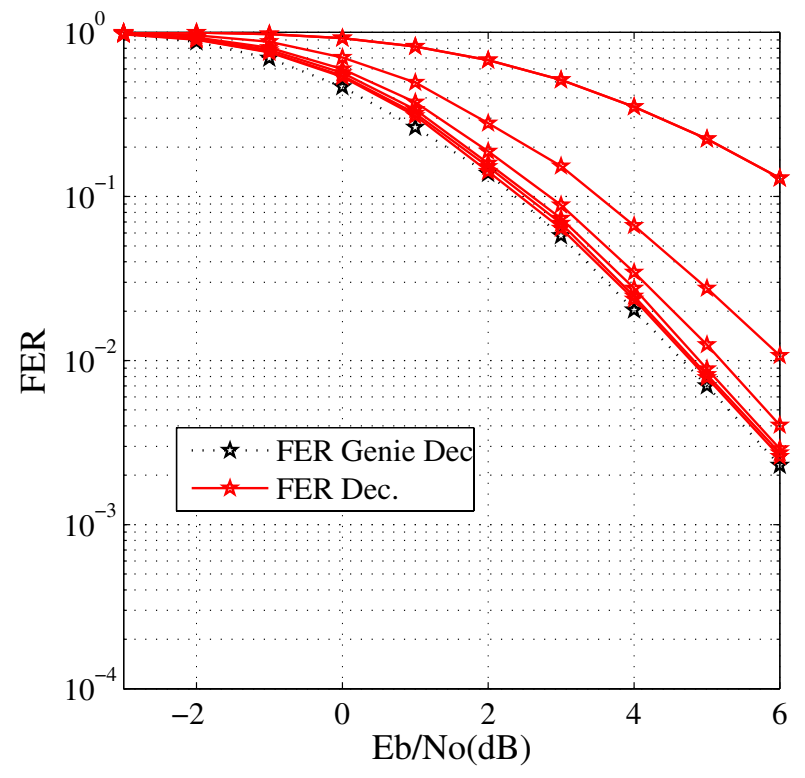

Figure 3. FER as a function of $\frac{E_{b}}{N_{0}}-2 \mathrm{Tx} 2 \mathrm{Rx}$ - CC(133,145,175)- 8-PSK

\subsection{Frequency-domain vs Time-domain MMSE Turbo equalization}

A further evaluation is performed to compare the performance of the proposed algorithm with that of the corresponding TD MMSE equalizer. Refering to [4, 17, 9], the TD MMSE equalizer consists of a soft interference canceler followed by a MMSE linear filter. It requires a sliding window, of sufficient length $N f$ from each antenna at the receiver to ensure optimum MSE solution. The issue is to find a value $N f$ that meets a compromise between performance and computation cost. The design of finite length filter in time-domain is well understood and finite length constraints can be considered under the MSE criterion [12]. We consider the same MIMO transmission scenario as in section IV. We define $r=\frac{\sigma_{\tilde{s}}^{2}}{\sigma_{s}^{2}}$ as the a priori information reliability degree. It can be seen that $r=1$ (resp. $r=0$ ), corresponds to perfect (resp. unavailable) a priori information from the decoder output. The deviation between the MSE of an equalizer using a $N f$ length impulse response filter and the minimum MSE is plotted as a function of $N f$ in Fig. 4 for different values of $r$ and $\frac{E_{b}}{N_{0}}$. As shown in Fig. 4, the filter length corresponding to the inflexion point in each curve, enables the equalizer to achieve a good compromise between complexity (short filter length) and ISI reduction (long filter length). Interestingly, we observe that the effective filter length decreases while the parameter $r$ increases. For $r=1$, the MMSE equalizer is able to retrieve the whole 


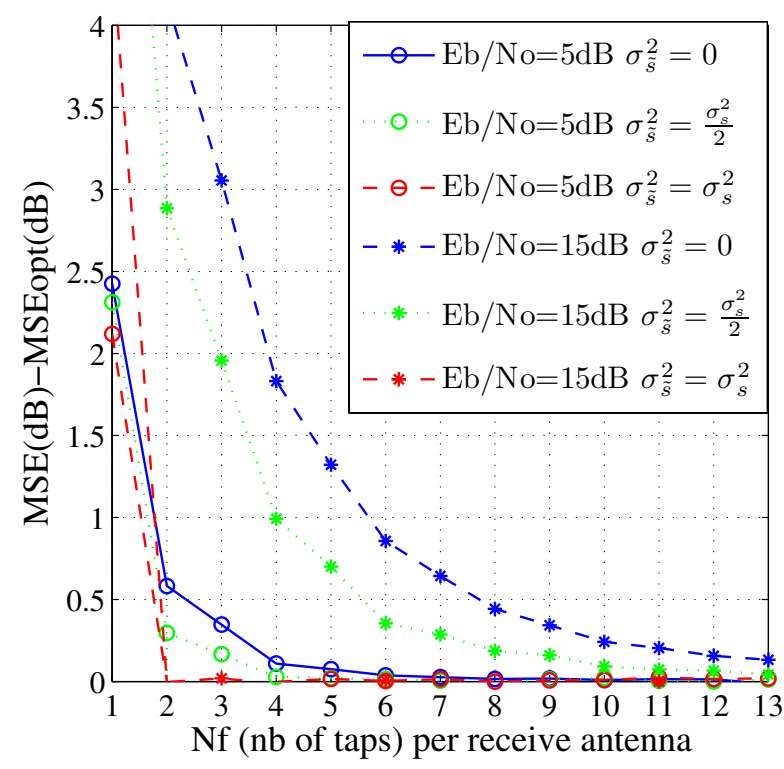

Figure 4. MSE as a function of $N f$ for various
reliability information levels $r=\sigma_{\tilde{s}}^{2} / \sigma_{s}^{2}-\frac{E_{b}}{N_{0}}=$
$5 \mathbf{d B}, 15 \mathbf{d B}-\mathbf{2}$ Tx 2 Rx - CC(133,145,175)- 8-
PSK

ISI from the received signal for each source to be estimated. In that case, the MMSE filter coincides with the matched filter. At high $\frac{E_{b}}{N_{0}}$ and $r \neq 1$, the ISI phenomenon dominates over the noise such that the equalizer has to invert the channel impulse response. As a consequence, a high impulse response length filter is necessary in order to avoid unstable phenomena due to finite length equalization constraints.

According to Fig. 4, using a $2 L+1$ length filter enables to achieve a good compromise between computational cost and performance for $\frac{E_{b}}{N_{0}}=5 \mathrm{~dB}$. We thus take $N f=2 L+1$ in the following. We consider the same MIMO transmission scenario as in section IV for $n_{T}=n_{R}=2$ with respectively $L=2$ and $L=10$ paths and variance $1 / L$. The bit error rate (resp. frame error rate) after the sixth decoding iteration is plotted as a function of $\frac{E_{b}}{N_{0}}$ in Fig. 5 (resp. Fig. 6). The performance of the turbo equalizer with perfect a priori information from the decoder is plotted as a reference.

We observe that the time-domain and frequency-domain techniques perform identically in each MIMO channel configuration when $N f=2 L+1$. We can conclude that the proposed MMSE turbo equalizer is the relevant frequencydomain counterpart of the time-domain. Note that, it is seen that the genie decoder performance corresponding to $L=2$ paths outperform the one respresented in Fig. 4.2 with a $1 \mathrm{~dB}$ gain which comes strictly from the improvement due to soft demapping with a priori knowledge. The figure also shows

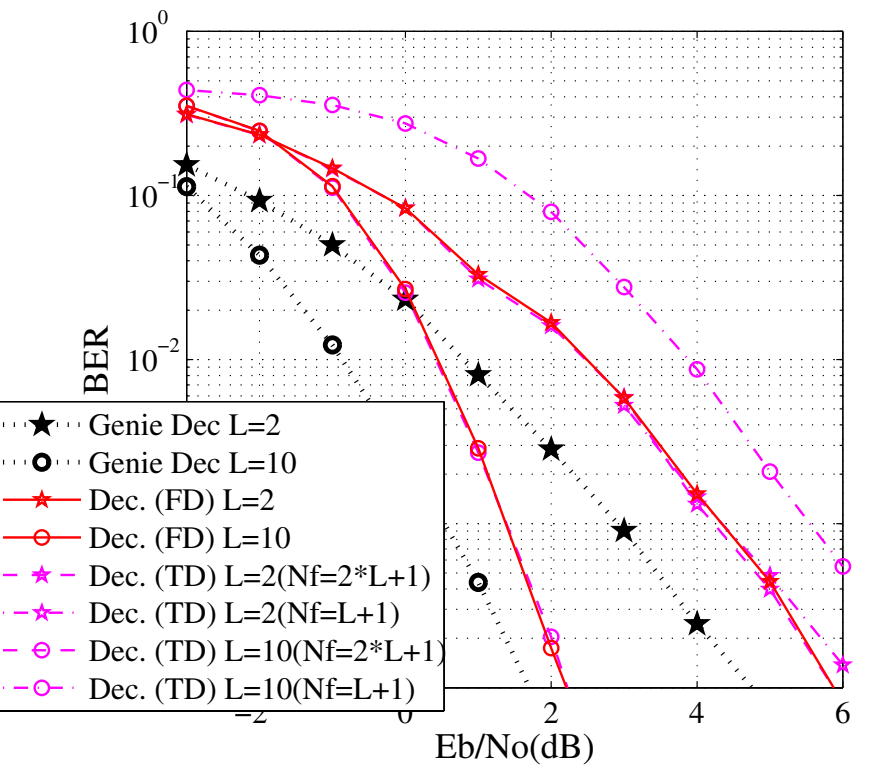

\section{Figure 5. BER as a function of $\frac{E_{b}}{N_{0}}-2$ Tx 2 Rx - CC(133,145,175)- 8-PSK. 6 iterations are performed}

performance improvement due to multipath diversity with the increase of channel impulse response length. Hence our system benefits from an additional diversity of order $L$ due to the channel dispersion.

We have also considered the TD MMSE turbo equalizer performance with a low number of filter coefficients $(L+1)$ to confirm the results in Fig. 4. As shown in Fig. 5 (resp. Fig. 6), the sixth decoding iteration corresponding to $L=2$ paths is very close to that of the TD turbo equalizer with sufficient filter length. It should be noted that if asymptotic performance is the same, the TD turbo equalizer with sufficient filter length exhibits faster convergence. On the other hand, noticeable loss in performance is observed for $L=10$ paths when the filter length is set to $L+1$. For $L=2$, taking $N f$ equal to 3 does not degrade the performance compared to using $N f=5$. On the contrary, for $L=10$, taking a 11-length filter brings a noticeable degradation in comparison with a 21-length filter. In Fig. 5, for a BER of $10^{-2}$ at the decoder output, there is a gap of $4 \mathrm{~dB}$ compared to the TD MMSE turbo equalizer with sufficient filter length. The same observations can be done in Fig. 6 for the FER.

Performing equalization in the time-domain requires a minimum filter length to achieve good performance at the expense of a high computational complexity for channels with severe ISI. In that case, the FD MMSE turbo equalizer seems more suitable. 


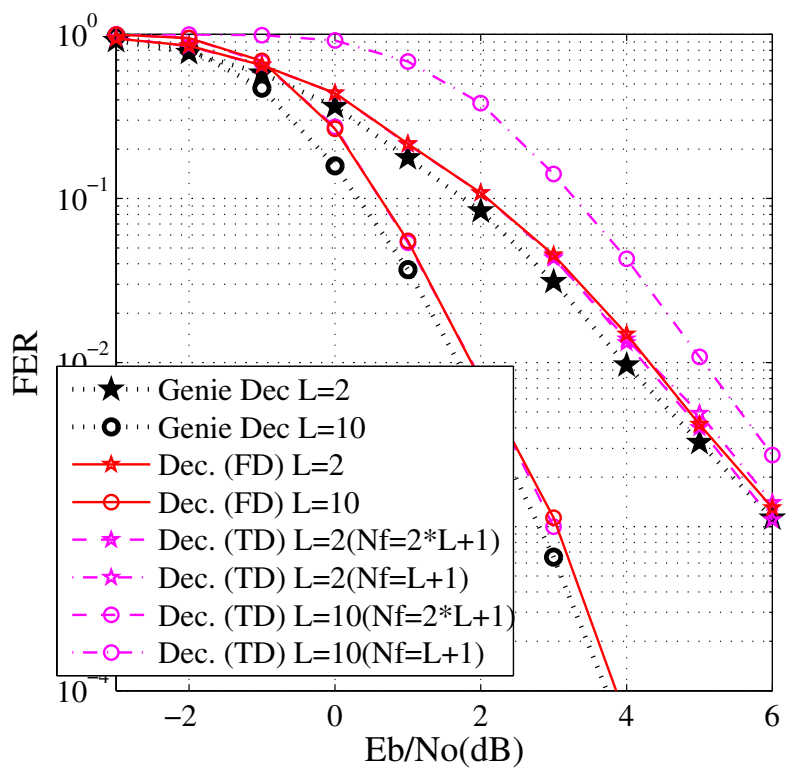

\section{Figure 6. FER as a function of $\frac{E_{b}}{N_{0}}-2 \mathrm{Tx} 2 \mathrm{Rx}$ - CC(133,145,175)- 8-PSK}

\section{Conclusions}

This paper describes a frequency domain turbo equalizer for Space Time Bit interleaved coded modulation. The proposed turbo equalizer differs from previous approaches in that feedforward and feedback filters are directly derived in the frequency domain. Asymptotic performance has been shown to be consistent with the probability of bit error derived in [11]. Simulations over multipath Rayleigh block fading channels show the structure equivalence in comparison with the time-domain approach. The frequency-domain equalizer structure is independent of the channel impulse response length on contrary of the time-domain one for which the filter length has to be properly chosen so as to ensure good performance. Future work will consider computation cost reduction of the whole iterative process in the case of TD receiver, by shortening the filter length with the iterations.

\section{References}

[1] T. Abe and T. Matsumoto. Space-Time Turbo Equalization in Frequency-Selective MIMO Channels. IEEE Trans. Veh. Tech., vol. 52, pp.469-475, May. 2003.

[2] N. Benvenuto and S. Tomasin. "On the comparison between OFDM ans Single Carrier modulation with a DFE using a frequency domain feedforward filter". IEEE Transactions on Communications, vol. 50 : pp. 947-955, June 2002.
[3] S. Chtourou, R. Visoz, and A. Berthet. "Low Complexity Frequency Domain Turbo Equalizer for STBICM over MIMO ISI Channel". Proc. ICECS'05, Gammarth, Tunisia, Dec. 2005.

[4] S. Chtourou, R. Visoz, and A. O. Berthet. A New Class of Iterative Equalizers for Space-Time BICM over MIMO Block Fading Multipath AWGN Channel. IEEE Trans. Commun., vol. 53, pp. 2076-2091, Dec. 2005.

[5] C. Douillard et. al. Iterative correction of inter symbol interference : Turbo equalization. European Trans. on Telecom., 6, pp. 507-511, Sep.-Oct.. 1995.

[6] A. Glavieux, C. Laot, and J. Labat. Turbo Equalization over a frequency selective channel. Proc. 1st Symp. Turbo Codes, Brest, France, pp. 96-102, Sept. 1997.

[7] C. S. K. Kansanen, T. Matsumoto, and R. Thomä. "Frequency-domain MMSE turbo equalization of multilevel coded QAM - Convergence in real fields". Proc. PIMRC 2005, Berlin, Germany, Sept. 2005.

[8] R. Kalbasi, R. Dinis, D. Falconer, and A. Banihashemi. "Layered Space-Time Receivers for Single-Carrier Transmission with Iterative Frequency-Domain Equalization". IEEE VTC 2004, Milan, Italy, May 2004.

[9] K. Kansanen, C. Schneider, T. Matsumoto, and R. Thoma. Multilevel-Coded QAM with MIMO Turbo-Equalization in Broadband Single-Carrier Signaling. Vehicular Technology Conf.Proceeding, 54(3), pp.954-966, May. 2005.

[10] C. Laot, R. Le Bidan, and D. Leroux. Low Complexity MMSE Turbo Equalization : a possible solution for EDGE. IEEE Trans. Commun., vol. 4 no. 3, pp. 965-974, May. 2005.

[11] N. Le Josse, C. Laot, and K. Amis. "Performance Validation for MMSE Turbo Equalization in ST-BICM Systems". IEEE VTC'06, Montréal, Canada, Sept. 2006.

[12] A. N. N. Al Dahir and A. Calderbank. "Finite Length MIMO Decision Feedback Equalization for Space Time Block Codes Signals over Multipath-fading Channels". IEEE Transactions on Vehicular Technology, vol. 10 : pp. 11761182, July 2001.

[13] A. Tonello. On Turbo Equalization of Interleaved SpaceTime Codes. VTC 2001., 2, pp.887-891, Oct. 2001.

[14] M. Tüchler and J. Hagenauer. "Linear time and frequency domain turbo equalization". IEEE VTC 2001, Rhodes, Greece, May 2001.

[15] M. Tüchler, A. Singer, and R. Koetter. Minimum mean squared error equalization using a priori information. IEEE Trans. Signal processing, 50(3), pp.673-683, Mar. 2002.

[16] X. Wang and H. Poor. Iterative (turbo) soft interference cancellation and decoding for coded CDMA. IEEE Trans. Commun., 47(7), pp.1046-1061, July. 1999.

[17] X. Wautelet, A. Dejonghe, and L. Vandendorpe. MMSEBased Turbo Receiver for Space-Time BICM Over Frequency-Selective MIMO Fading Channels. IEEE Trans. Signal Processing, 52(6), pp.1804-1809, June. 2004.

[18] M. Yee, M. Sandell, and Y. Sun. Comparison study of Single-carrier and Multi-carrier modulation using iterative based receiver for MIMO system. VTC 2004, 3, pp. 12751279, May. 2004. 